\title{
Kohlenstoff und Klimawandel Neue Herausforderungen für das Risikomanagement von Unternehmen
}

\section{Journal Article}

Author(s):

Hoffmann, Volker H.; Busch, Timo

Publication date:

2008

Permanent link:

https://doi.org/10.3929/ethz-b-000011547

Rights / license:

In Copyright - Non-Commercial Use Permitted

Originally published in:

UmweltWirtschaftsForum 16(2), https://doi.org/10.1007/s00550-008-0072-5 


\title{
Kohlenstoff und Klimawandel - neue Herausforderungen für das Risikomanagement von Unternehmen
}

\author{
Volker H. Hoffmann • Timo Busch
}

Online veröffentlicht: 24.4.2008

(C) Springer-Verlag 2008

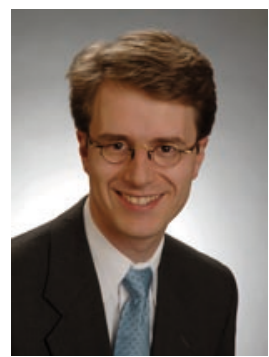

Volker H. Hoffmann

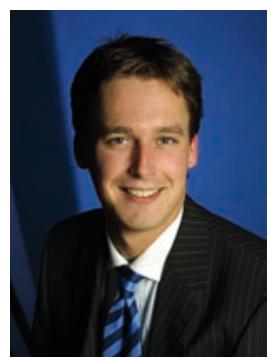

Timo Busch

Zusammenfassung Die Verfügbarkeit fossiler Ressourcen und der Klimawandel verändern die Rahmenbedingungen des Wirtschaftens. Dieser Beitrag skizziert sich daraus ergebende „Carbon Constraints“ für Unternehmen und erläutert Ansatzpunkte für betriebliche Strategien zum Risikomanagement.

\section{Einleitung}

Die globale Wirtschaft sieht sich mit zwei zentralen energiebezogenen Herausforderungen konfrontiert, der eingeschränkten Verfügbarkeit von Energieressourcen einerseits und den ökologischen Konsequenzen eines zu hohen Energieverbrauchs andererseits (IEA, 2006). Bei einer Betrachtung der Inputdimension lässt sich eine Änderung der wirtschaftlichen Rahmenbedingungen am Beispiel Öl zeigen: Die maximale Menge an Öl, die in einem Jahr weltweit gefördert werden kann, wird als „Peak Oil““ bezeichnet.

Volker H. Hoffmann ( $\square$ ), Timo Busch

ETH Zürich, Department for Management, Technology and Economics Kreuzplatz 5, 8032 Zürich, Schweiz

E-Mail: vhoffmann@ethz.ch
Nachdem dieser Punkt überschritten ist, nimmt die jährliche Ölproduktion ab. Wissenschaftliche Schätzungen zum Peak Oil variieren: Während z. B. Bentley (2002) erwartet, dass der Peak zwischen 2007 und 2012 erreicht sein wird, geht Campbell (2005) davon aus, dass er bereits 2004 überschritten wurde. Parallel dazu steigt die Ölnachfrage vor allem aufgrund der zunehmenden Industrialisierung von Staaten wie China und Indien kontinuierlich an. Angesichts dieser sich öffnenden Schere zwischen Angebot und Nachfrage dürften die Rohölpreise, abgesehen von kurzfristigen Schwankungen aufgrund tagespolitischer Ereignisse, langfristig deutlich ansteigen (IEA, 2006; Reynolds, 1999) und Kostensteigerungen insbesondere für Unternehmen in kohlenstoff- und energieintensiven Branchen mit sich bringen.

Auch in der Outputdimension, der globalen Klimaänderung als Konsequenz der Emission von Treibhausgasen (IPCC, 2007), wird deutlich, dass sich die wirtschaftlichen Rahmenbedingungen ändern. Der von der britischen Regierung in Auftrag gegebene Stern (2006) Report beziffert durch den Klimawandel bedingte mögliche Wohlstandseinbussen auf bis zu $20 \%$ des weltweiten Sozialprodukts, falls keine Gegenmaßnahmen ergriffen werden. Eine politische Gegenmaßnahme stellt z.B. der Plan der EU dar, die $\mathrm{CO}_{2}$ Emissionen bis 2020 um 20\% relativ zu 1990 zu senken und eine Dekarbonisierung der Gesellschaft um 60-80\% bis 2050 anzustreben (European Commission, 2007). Als Folge dieser Entwicklungen sind Unternehmen nicht nur mit direkten Effekten des Klimawandels wie z. B. einer $\mathrm{Zu}$ nahme wetterbedingter Schäden konfrontiert, sondern auch mit indirekten Effekten wie gesetzlichen Maßnahmen zur Reduktion der Treibhausgas-Emissionen.

In der Vergangenheit wurde die natürliche Umwelt in der internationalen Managementliteratur oft konzeptionell vernachlässigt und als irrelevant für den Geschäftsbetrieb angesehen (Winn \& Kirchgeorg, 2005). Die obigen Bei- 
spiele verdeutlichen aber, dass ein Umdenken nötig ist: Die generelle Verfügbarkeit fossiler Ressourcen und die unbeschränkte Emission von Treibhausgasen können nicht länger als statische Rahmenbedingungen des Wirtschaftens angesehen werden. Stattdessen ist aus ressourcenökonomischer wie gesellschaftspolitischer Perspektive eine zunehmende Dynamik zu beobachten, die sich in Zukunft weiter verstärken dürfte. Die geschilderten kohlenstoff-bezogenen Änderungen der wirtschaftlichen Rahmenbedingungen können den Handlungsspielraum von Unternehmen einschränken und werden dementsprechend als „Carbon Constraints“ bezeichnet (Busch \& Hoffmann, 2007).

Carbon Constraints können sowohl in der In- als auch in der Outputdimension erhebliche Risiken für Unternehmen oder ganze Branchen darstellen, z. B. wenn sich klimatische Bedingungen ändern oder wenn durch die Bepreisung von Treibhausgas-Emissionen etablierte Produktionsprozesse innerhalb von Wertschöpfungsketten unrentabel werden. Das betriebliche Risikomanagement muss einen adäquaten Umgang mit diesen Risiken gewährleisten, sieht sich dabei allerdings mit erschwerenden Aspekten konfrontiert: Ausmaß und Eintrittswahrscheinlichkeit der Carbon Constraints sind ungewiss, aufgrund der komplexen globalen Zusammenhänge ist ein Vergleich mit traditionellen Risiken nur eingeschränkt möglich und spezifische Effekte können nur bedingt zu Einzelunternehmen zugeordnet werden.

\section{Carbon Constraints - neue Risiken in zwei Dimension}

Grundsätzlich interagiert eine Organisation mit der natürlichen Umwelt in zweierlei Hinsicht (vgl. Haigh \& Griffiths, 2007;
Winn \& Kirchgeorg, 2005): Einerseits nutzt sie Ressourcen aus der Umwelt und gibt - gegebenenfalls abgewandelte Ressourcen wieder in diese zurück. Dieser Prozess kann mehr oder weniger schädlich für die Umwelt sein. Andererseits ist jede Organisation in die natürliche Umwelt eingebettet. Ändert sich diese Umwelt, können mehr oder weniger negative Effekte für die Organisation auftreten. Diese allgemeinen Überlegungen können auf Unternehmen und Carbon Constraints übertragen werden, um mögliche Risikoprofile einzuschätzen und daraus betriebliche Strategien zum Risikomanagement abzuleiten. Dabei ergeben sich zwei Dimensionen.

Betriebliche Risikomanagementstrategien, die versuchen die Knappheit fossiler Ressourcen sowie indirekte Effekte des Klimawandels wie z. B. die Auswirkungen eines Emissionshandels zu adressieren, setzen jeweils bei der Frage an, wie viel Kohlenstoff in einem Unternehmen genutzt wird. Daher kann für eine systematische Darstellung von Risikoprofilen im Kontext von Carbon Constraints eine Dimension „Ausmaß der betrieblichen Kohlenstoffnutzung" entwickelt werden.

Die zweite Dimension beschreibt die Konsequenzen für ein Unternehmen, die sich aus der Veränderung der natürlichen Umwelt ergeben, hervorgerufen durch die nicht nachhaltige Nutzung fossiler Ressourcen und die Emission von Treibhausgasen. Während hierunter auch mit der Ressourcengewinnung verbundene Aspekte fallen wie z.B. tektonische Instabilitäten in Abbaugebieten, fokussiert die weitere Darstellung auf den aus Risikoperspektive wichtigeren Klimawandel. Die zweite Dimension beschreibt daher die „Klimawandel-Exponiertheit des Unternehmens“. Aus der Kombination der beiden Dimensionen resultiert dann eine Matrix, die das mögliche Ausmaß von Carbon Constraints für ein Unternehmen veranschaulicht (siehe Abbildung 1).
Abb. 1 Risikoprofile von Unternehmen gegenüber Carbon Constraints

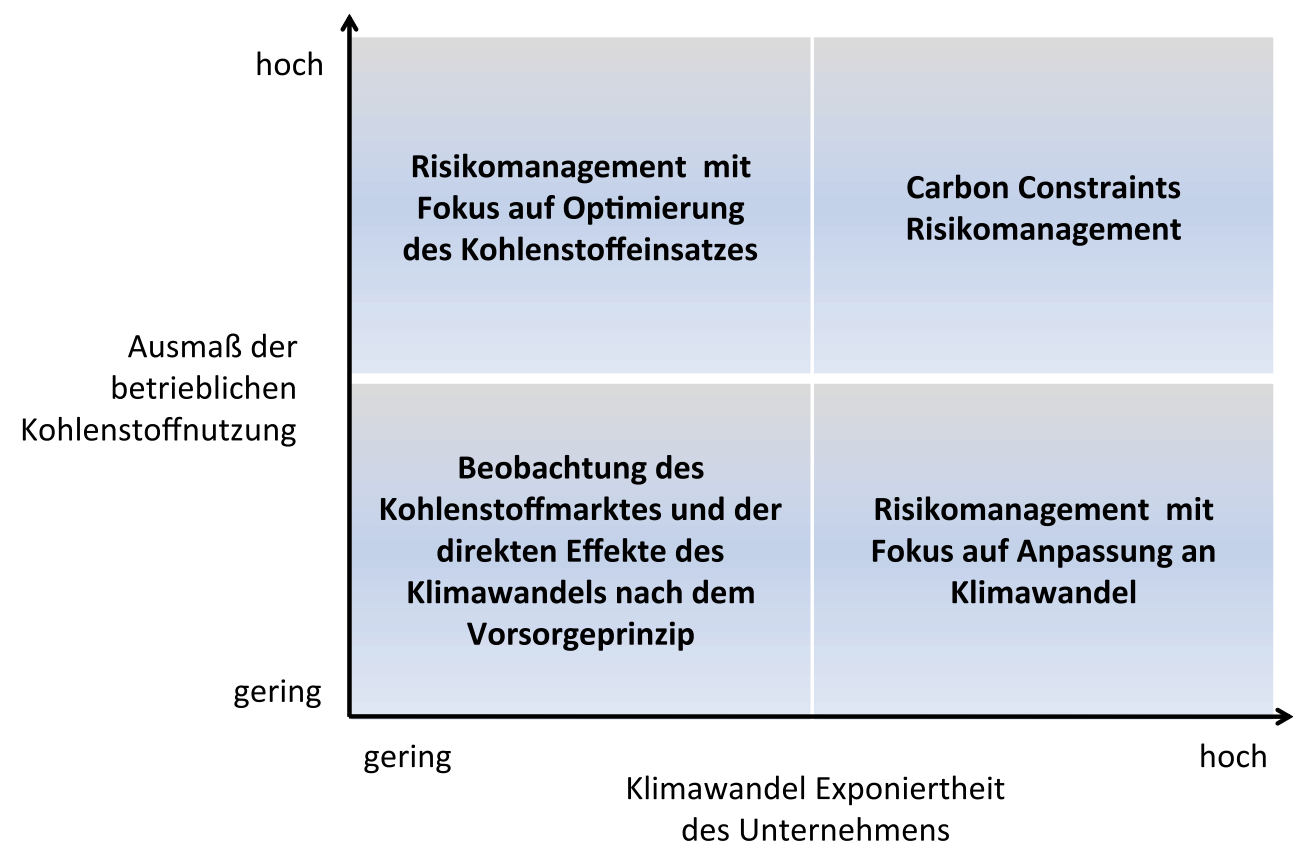


Durch die Einteilung in diese $2 \times 2$ Matrix ergeben sich vier generische Risikoprofile, die durch unterschiedliche Schwerpunkte im Risikomanagement adressiert werden können: Unternehmen mit hoher Kohlenstoffnutzung, aber geringer Exponiertheit gegenüber Klimawandel (oben links) sollten einen Fokus auf die Optimierung ihres Kohlenstoffeinsatzes legen. Dabei muss allerdings die ganze Wertschöpfungskette betrachtet werden, da kohlenstoff-bedingte Risiken auch bei Zulieferern oder Kunden lauern können. Dies zeigt etwa das Beispiel der Automobilindustrie, bei der eine Optimierung des Kohlenstoffeinsatzes insbesondere bei der Nutzungsphase ansetzen sollte. Demgegenüber sollten Unternehmen mit hoher Exponiertheit, aber geringer Kohlenstoffnutzung (unten rechts) primär Anpassungsstrategien an den Klimawandel entwickeln. Dies ist beispielsweise der Fall für Agro-Unternehmen, die sich wetterbedingt mit einer Beeinträchtigung ihrer bisherigen landwirtschaftlichen Nutzung konfrontiert sehen. Unternehmen, für die beide Bereiche gleichsam relevant sind (oben rechts) benötigen ein umfassendes Risikomanagement, das alle Bereiche der Carbon Constraints adressiert. Dies trifft z. B. auf Stromerzeuger mit einem hohen Anteil an Kohle-basierter Erzeugung zu, wenn Kraftwerke an Flüssen liegen, die bei längeren Trockenperioden einen zu niedrigen Wasserspiegel zur Kühlung der Kraftwerke aufweisen könnten. Unten links befinden sich Unternehmen, die unter derzeitigen Rahmenbedingungen weder aufgrund ihrer Kohlenstoffnutzung noch hinsichtlich ihrer Klimawandel-Exponiertheit ein spezifisches Risikomanagement benötigen, aber beide Dimensionen nach dem Vorsorgeprinzip beobachten sollten. Ein Beispiel hierfür ist der Einzelhandel. Zwar ist derzeit keine massive Beeinträchtigung des Geschäftsbetriebs durch eine der beiden Dimensionen absehbar. Allerdings sollten Unternehmen den Einfluss von Carbon Constraints unter anderem auf die erforderlichen Transporte oder die Produktvielfalt kritisch beobachten. Im Folgenden werden Ansätze aufgezeigt, wie Unternehmen ihre Positionierung im Hinblick auf die beiden Dimensionen abschätzen können.

\section{Bewertung der betrieblichen Kohlenstoffnutzung}

Das Ausmaß der betrieblichen Kohlenstoffnutzung kann mit Carbon Performance Indikatoren bestimmt werden. Solche Indikatoren müssen zunächst die absolute Höhe der Kohlenstoffnutzung berücksichtigen, indem die inner- und außerbetrieblichen Kohlenstoffflüsse ${ }^{1}$ systematisch analysiert werden.

Da sich verschiedene Risiken auch aus der Kohlenstoffnutzung von Zulieferern oder Kunden ergeben können, ist eine Betrachtung unter Einbezug der Wertschöpfungskette nötig. Für eine emissionsorientierte Darstellungsweise der Kohlenstoffflüsse hat die Greenhouse Gas Protocol Initiative (WBCSD \& WRI, 2004) ein Klassifikationsschema entwickelt, das drei Bilanzrahmen, sogenannte "Scopes“, beinhaltet. Scope 1 umfasst alle direkten TreibhausgasEmissionen eines Unternehmens, die z. B. bei der Verbrennung von Heizöl anfallen. Im Scope 2 werden indirekte Treibhausgas-Emissionen betrachtet, die außerhalb des eigenen Unternehmens während der Energiebereitstellung (Elektrizität, Wärme, Dampf) entstehen. Bezieht z. B. ein Unternehmen Strom von einem Kohlekraftwerk, so sind die bei der Stromerzeugung entstehenden Emissionen Teil der indirekten Emissionen des Unternehmens. Scope 3 beinhaltet alle weiteren vor- und nachgelagerten indirekten Treibhausgas-Emissionen. Bei Automobilherstellern sind dies z.B. die - schwer abzuschätzenden - Emissionen während der Nutzungsphase des Autos.

Abbildung 2 veranschaulicht, dass die Darstellung der Kohlenstoffflüsse - analog der Greenhouse Gas Protocol Initiative - auch mit Hilfe einer inputorientierten Darstellungsweise erfolgen kann. Der Vorteil dieser Darstellung

\footnotetext{
${ }^{1}$ Obwohl nicht alle im Kyoto-Protokoll aufgeführten Treibhausgase (THG) Kohlenstoff beinhalten, werden hier alle THG unter der Verwendung von $\mathrm{CO}_{2}$-Äquivalenten als Kohlenstoff Outputs subsummiert. Allerdings ist zu beachten, dass $\mathrm{CO}_{2}$ deren Hauptbestandteil darstellt: Beispielsweise sind durchschnittlich $93 \%$ der THG Emissionen der FTSE 100 Unternehmen $\mathrm{CO}_{2}$-bezogen (Henderson \& Trucost, 2005).
}

Abb. 2 Kohlenstoff In- und Outputs entlang der Wertschöpfungskette
Kohlenstoff Inputs: Fossile Ressourcen

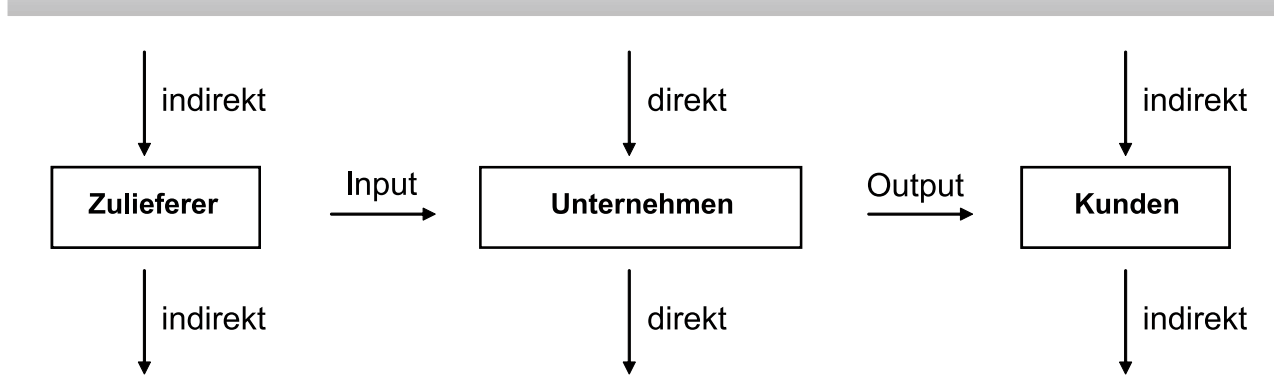

Kohlenstoff Outputs: Treibhausgas-Emissionen 
kann darin liegen, dass sich die entsprechenden Verbrauchsmengen mit Hilfe betrieblicher Informationssysteme einfacher erheben lassen. Ein Nachteil dieser Darstellungsweise ergibt sich dadurch, dass z. B. Maßnahmen wie das Offsetting von $\mathrm{CO}_{2}$-Emissionen oder die $\mathrm{CO}_{2}$-Speicherung (Carbon Capture and Storage) zu keiner Ergebnisveränderung führen und nicht alle treibhausgasrelevanten Inputs erfasst werden.

Zusätzlich muss die Kohlenstoffnutzung eines Unternehmens in den betriebswirtschaftlichen Kontext gesetzt werden, um Veränderungen über Zeit (z. B. Wachstum, M\&A) $\mathrm{zu}$ berücksichtigen und Vergleiche (z. B. Aussagen über Verbesserungsmaßnahmen) zu ermöglichen. Die absolute Menge der Kohlenstoffnutzung (Zähler) kann dazu ins Verhältnis zu einer betriebswirtschaftlichen Kennzahl (Nenner) gesetzt werden. Je nach Zielsetzung der Analyse existieren dabei verschiedene Darstellungsmöglichkeiten für den Nenner wie etwa Umsatz, EBITDA, Marktkapitalisierung oder Produktionsmengen (Henderson \& Trucost, 2005; Holcim, 2006; Hutchinson, 2006). ${ }^{2}$

Als Resultat ergibt sich die Kohlenstoff-Intensität als ein Maß, mit dem Unternehmen ihre Sensitivität hinsichtlich der zuvor beschriebenen Risiken bestimmen können. Beispielsweise kann mit Hilfe der Kohlenstoff-Intensität gezeigt werden, wie viel Treibhausgas-Emissionen ein Unternehmen pro EUR Umsatz emittiert. Unter Berücksichtigung eines Kohlenstoffpreises (z. B. basierend auf Zertifikaten des Europäischen Emissionshandelssystems) kann so das emissionsbedingte Risiko pro EUR Umsatz abgeschätzt oder ein potentieller $\mathrm{CO}_{2}$-Risikozuschlag berechnet werden.

\section{Analyse der Klimawandel-Exponiertheit des Unternehmens}

Bei der Analyse, wie stark ein Unternehmen gegenüber dem Klimawandel exponiert ist, muss zwischen zwei Entwicklungen unterschieden werden, die im Zusammenhang mit der globalen Klimaänderung stehen (Romilly, 2007). Einerseits hängt die Exponiertheit stark von der Zunahme von ad hoc auftretenden Einzelereignissen wie Stürmen oder Überschwemmungen ab, die zu Beeinträchtigungen und materiellen Schäden führen können. Rückversicherer beobachten schon seit einiger Zeit eine Zunahme solcher extremen Wetterereignisse und der damit einhergehenden volkswirtschaft-

\footnotetext{
${ }^{2}$ Es ist wichtig, genau auf die Aussagekraft der gewählten Basis zu achten: So birgt die Verwendung des Umsatzes in nominalen Werten die Gefahr, dass Verbesserungen allein durch die natürliche Inflation bedingt sind. Die Verwendung der Marktkapitalisierung hat den Nachteil, dass durch M\&A Aktivitäten die Ergebnisse sich auf unterschiedliche Bezugsrahmen beziehen. Kennziffern, die auf der EBITDA Marge beruhen, unterliegen oft Schwankungen durch Änderungen im Geschäftsgang.
}

lichen Schäden (Heck, Bresch, \& Tröber, 2006). Andererseits wird die Klimawandel-Exponiertheit durch langsame, mit der Klimaänderung einhergehende Entwicklungen beeinflusst wie z.B. dem Anstieg der durchschnittlichen Temperatur oder des Meeresspiegels. Bei solchen eher kontinuierlichen Ereignissen lässt sich eine Veränderung und somit eine Zunahme der Exponiertheit nur langfristig bestimmen.

Für die Risiko-Analyse besteht ein elementarer Unterschied zwischen den beiden Entwicklungen: Die Zunahme bei Extremereignissen kann zwar als ein statistischer Anstieg der Eintrittswahrscheinlichkeit der Ereignisse abgebildet werden, z. B. indem regionale Risikoprofile erstellt werden (Romilly, 2007). Allerdings sind solche Ereignisse bezogen auf den Einzelfall (z. B. die Stärke eines Sturms im nächsten Jahr in einer bestimmten Region) nur schwer prognostizierbar (Smit, Burton, Klein, \& Wandel, 2000). Potentielle Schäden für ein Unternehmen können jedoch in den meisten Fällen grob abgeschätzt werden (z. B. anhand des absehbaren Produktionsausfalls). Ganz anders verhält sich dies bei langfristigen Effekten der Klimaänderung. Basierend auf Zeitreihenanalysen lassen sich zwar Aussagen über mögliche zukünftige Entwicklungen treffen. Allerdings kann weniger genau abgeschätzt werden, welche monetären Konsequenzen sich hieraus für ein einzelnes Unternehmen ergeben.

Neben diesen generellen Unterschieden hinsichtlich der Bestimmung von Eintrittswahrscheinlichkeiten und Schadensabschätzungen bestehen ferner Unterschiede hinsichtlich der Sensitivität und Anpassungsfähigkeit von verschiedenen Industrien und Unternehmen innerhalb einer Industrie (Burton, 1996). So sind auf der einen Seite manche Industrien stärker als andere von langsam zunehmenden Temperaturen betroffen (vgl. süddeutsche Skigebiete vs. Weinbauern im Rhein-Main Gebiet). Auf der anderen Seite können Unternehmen innerhalb einer Industrie abhängig von ihrer geographischen Ansiedlung individuell unterschiedlich von Naturextremen wie z. B. Fluten oder Stürmen betroffen sein (vgl. Produktionsstätte in einem von Überschwemmungen gefährdeten Gebiet vs. deutsches Mittelgebirge).

Es wird deutlich, dass sich diese potentiellen Entwicklungen nicht gleichermaßen wie die Kohlenstoffnutzung anhand von einfachen Kennzahlen ausdrücken und in Bezug zu betrieblichen Größen setzen lassen. Vielmehr muss jedes Unternehmen seine potentiellen Risiken aufgrund der spezifischen Klimawandel-Exponiertheit mit Hilfe qualitativer oder semi-quantitativer Analysen bestimmen. Folgende Schritte können dabei als Hilfestellung dienen (vgl. Berkhout, Hertin, \& Gann, 2006): (1) Wie exponiert ist die Industrie gegenüber der globalen Klimaänderung und extremen Wetterereignissen? (2) Ist das Unternehmen darüber hinaus gegenüber der globalen Klimaänderung und extremen Wetterereignissen besonders exponiert? (3) Was sind mögliche zukünftige Entwicklungen für die Exponiertheit der Industrie und des Unternehmens? 
Abb. 3 Risikomanagementstrategien als Antworten auf Carbon Constraints

\begin{tabular}{|c|c|c|c|c|}
\hline \multicolumn{2}{|c|}{ Strategie fokussiert auf } & \multirow{2}{*}{$\begin{array}{l}\text { Risiko } \\
\text { Reduktion } \\
\text { Effizienz fossiler } \\
\text { Ressourcen } \\
\text { steigern }\end{array}$} & \multirow{2}{*}{$\begin{array}{l}\text { Risiko } \\
\text { Kompensation } \\
\text { Preis für fossile } \\
\text { Ressourcen } \\
\text { absichern }\end{array}$} & \multirow{2}{*}{$\begin{array}{l}\text { Risiko } \\
\text { Vermeidung } \\
\text { Fossile } \\
\text { Ressourcen } \\
\text { substituieren }\end{array}$} \\
\hline $\begin{array}{l}\text { Optimierung } \\
\text { des }\end{array}$ & $\begin{array}{l}\text { Kohlenstoff } \\
\text { Inputs }\end{array}$ & & & \\
\hline $\begin{array}{l}\text { Kohlenstoff- } \\
\text { einsatzes }\end{array}$ & $\begin{array}{l}\text { Kohlenstoff } \\
\text { Outputs }\end{array}$ & $\begin{array}{l}\text { Carbon capture } \\
\& \text { storage } \\
\text { nutzen }\end{array}$ & $\begin{array}{l}\text { Finanzderivate für } \\
\text { Emissionsrechte } \\
\text { kaufen }\end{array}$ & $\begin{array}{l}\text { In Region ohne } \\
\text { Klimaregulierung } \\
\text { ausweichen }\end{array}$ \\
\hline \multirow[t]{2}{*}{$\begin{array}{l}\text { Anpassung an } \\
\text { Klimawandel }\end{array}$} & $\begin{array}{l}\text { Extreme } \\
\text { Wetter- } \\
\text { ereignisse }\end{array}$ & $\begin{array}{l}\text { Schadens- } \\
\text { anfälligkeit } \\
\text { senken }\end{array}$ & $\begin{array}{l}\text { Potentielle } \\
\text { Schäden } \\
\text { versichern }\end{array}$ & $\begin{array}{l}\text { Produktions- } \\
\text { stätten verlagern }\end{array}$ \\
\hline & $\begin{array}{l}\text { Stetiger } \\
\text { Temperatur- } \\
\text { anstieg }\end{array}$ & $\begin{array}{l}\text { Temperatur- } \\
\text { Abhängigkeit } \\
\text { minimieren }\end{array}$ & $\begin{array}{l}\text { Lokale Gegen- } \\
\text { maßnahmen } \\
\text { ergreifen }\end{array}$ & $\begin{array}{l}\text { Geschäftsmodell } \\
\text { abändern }\end{array}$ \\
\hline
\end{tabular}

\section{Unternehmerische Antwortoptionen auf Carbon Constraints}

Für beide oben beschriebenen Risikoprofile, „Optimierung des Kohlenstoffeinsatzes“ und „Anpassung an Klimawandel“" (vgl. Abbildung 1) können drei grundlegende, miteinander kombinierbare Antwortstrategien unterschieden werden, für die Abbildung 3 Beispiele veranschaulicht.

Bei Risiko-Reduktions-Strategien wird das Ziel verfolgt, das Risiko durch gezielte Maßnahmen zu minimieren, die Risikoquelle aber nicht vollständig zu eliminieren. Dies ist z. B. der Fall, wenn ein Unternehmen durch betriebliche Effizienzsteigerungen den Verbrauch an fossilen Ressourcen reduziert und dadurch den Risiken von Preisschwankungen weniger ausgesetzt ist.

Bei Risiko-Kompensations-Strategien wird versucht, die Auswirkung des Risikos auf das Unternehmen zu mindern, indem Risiken z.B. externalisiert werden. Dies geschieht etwa, wenn ein Unternehmen die mit schwankenden Preisen für Emissionsrechte verbundenen Risiken durch den Kauf von Finanzderivaten auf Emissionsrechte absichert.

Bei Risiko-Vermeidungs-Strategien versucht das Unternehmen, dem Risiko auszuweichen. Hierfür müssen der Ursprung des Risikos bekannt sein und Alternativen existieren. Dann besteht gegebenenfalls die Möglichkeit, vollständig unabhängig von bestimmten Carbon Constraints Risiken zu werden. Dies ist etwa der Fall, wenn durch die Verlagerung von Produktionsstätten in weniger von extremen Wetterereignissen betroffene Gebiete das Risiko bestimmter materieller Schäden umgangen werden kann (Burton, 1996).

\section{Diskussion}

Carbon Constraints stellen bereits heute für viele Unternehmen finanziell relevante Aspekte dar, die in Zukunft noch wichtiger werden dürften. Deutlich wird dies z. B. durch den
Anstieg des Ölpreises, das Inkrafttreten der zweiten Phase des Europäischen Emissionshandelssystems oder die $\mathrm{Zu}$ nahme an wetterbedingten Schäden.

Die Berücksichtigung von Carbon Constraints wird damit zu einem wichtigen betrieblichen Erfolgsfaktor, und betroffene Unternehmen sollten aufgrund der Zukunfts-Relevanz der Themen ein systematisches Risikomanagement etablieren. Hierfür sind vier Schritte elementar: (1) Unternehmen sollten ihr Risikoverständnis um die Aspekte Ausmaß der betrieblichen Kohlenstoffnutzung sowie der Exponiertheit gegenüber Klimawandel erweitern. (2) Beide Aspekte sollten in bestehende Risikosysteme integriert und analog $\mathrm{zu}$ anderen betrieblichen Risiken erfasst werden. (3) Um die zeitlichen Veränderungen von Carbon Constraints adäquat abzubilden, bedarf es eines entsprechenden Risikocontrollings. (4) Sowohl die Erfassung der Risiken als auch deren Veränderung sollte eng mit dem strategischen Management verknüpft werden, um eine frühzeitige Berücksichtigung in der Unternehmensstrategie zu ermöglichen.

Zukünftige Forschungsarbeiten können die in diesem Beitrag diskutierten Aspekte vertiefend analysieren. Beispielsweise können quantitative Analysen die sich aus dem Ausmaß der Kohlenstoffnutzung oder der Exponiertheit gegenüber dem Klimawandel ergebenden Risiken ins Verhältnis zur finanziellen Performance von Unternehmen setzen. Dies dürfte insbesondere für Vergleiche innerhalb einer Industrie interessant sein, um solche Informationen für Best-in-Class Bewertungen $\mathrm{zu}$ verwenden. Ferner könnte mit Hilfe von qualitativen empirischen Analysen dargestellt werden, wie sich die vier Risikoprofile detailliert beschreiben lassen und wie unternehmerische Best-Practice Ansätze für ein gutes Carbon Constraints Risikomanagement aussehen können.

\section{Literatur}

Bentley R W (2002) Global oil \& gas depletion: an overview. Energy Policy 30(3):189-205 
Berkhout F, Hertin J, Gann DM (2006) Learning to adapt: Organisational adaptation to climate change impacts. Climatic Change 78(1):135-156

Burton I (1996) The Growth of Adaptation Capacity: Practice and Policy. In: Smith J, Bhatti N, Menzhulin G, Benioff R, Budyko MI, Campos M, Jallow B, Rijsberman F (Eds) Adapting to Climate Change: An International Perspective: 55-67. New York: Springer

Busch T, Hoffmann VH (2007) Emerging carbon constraints for corporate risk management. Ecological Economics, 62(3-4):518-528

Campbell, CJ (2005) Oil Crisis. Multi-Science. Brentwood: MultiScience Publishing

European Commission (2007) EU action against climate change, Office for Official Publications of the European Communities. Brussels

Haigh N, Griffiths A (2007) The Natural Environment as a Primary Stakeholder: the Case of Climate Change. Business Strategy and the Environment, (in press)

Heck P, Bresch D, Tröber S (2006) The effects of climate change: Storm damage in Europe on the rise. Zurich: Swiss Reinsurance Company

Henderson \& Trucost (2005) The Carbon 100 - Quantifying the Carbon Emissions, Intensities and Exposures of the FTSE 100. London: Henderson Global Investors

Holcim (2006) Corporate Sustainable Development Report 2005. Zurich
Hutchinson H (2006) Climate change: when hell freezes over - European utilities. London: ING

IEA (2006) World Energy Outlook 2006, International Energy Agency. Paris

IPCC (2007) Climate Change 2007: The Physical Science Basis. Cambridge: Cambridge University Press

Reynolds DB (1999) The mineral economy: how prices and costs can falsely signal decreasing scarcity. Ecological Economics $31: 155-166$

Romilly P (2007) Business and climate change risk: a regional time series analysis. Journal of International Business Studies $38: 474-480$

Smit B, Burton I, Klein RJT, Wandel J (2000) An anatomy of adaptation to climate change and variability. Climatic Change 45(1): 223-251

Stern N (2006) The Economics of Climate Change - The Stern Review. Cambridge: Cambridge University Press

WBCSD \& WRI (2004) The Greenhouse Gas Protocol: A Corporate Accounting and Reporting Standard (Revised version), World Business Council for Sustainable Development, World Resources Institute. Geneva, Washington DC

Winn M, Kirchgeorg M (2005) The siesta is over: a rude awakening from sustainability myopia. In: Sharma S, Starik M (Eds), Research in Corporate Sustainability, Volume 3, Strategic Capabilities and Competitiveness: 232-258. Northampton: Elgar 